\title{
Multiple Sources of Solar High-energy Protons
}

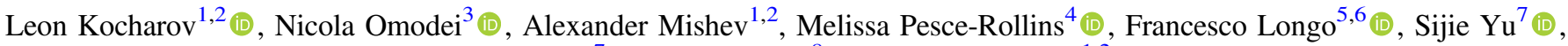 \\ Dale E. Gary ${ }^{7}$ (D), Rami Vainio ${ }^{8}$ (D), and Ilya Usoskin ${ }^{1,2}$ (ID) \\ ${ }^{1}$ Space Physics and Astronomy Research Unit, Faculty of Sciences, P.O.B. 3000, Oulu FI-90014, Finland \\ ${ }^{2}$ Sodankylä Geophysical Observatory, University of Oulu, Oulu FI-90014, Finland \\ ${ }^{3}$ W.W. Hansen Experimental Physics Laboratory, Kavli Institute for Particle Astrophysics and Cosmology, Department of Physics and SLAC National Accelerator \\ Laboratory, Stanford University, Stanford, CA 94305, USA \\ ${ }^{4}$ Istituto Nazionale di Fisica Nucleare, Sezione di Pisa, I-56127 Pisa, Italy \\ ${ }^{5}$ Istituto Nazionale di Fisica Nucleare, Sezione di Trieste, I-34127 Trieste, Italy \\ ${ }^{6}$ Dipartimento di Fisica, Università di Trieste, I-34127 Trieste, Italy \\ ${ }^{7}$ Center for Solar-Terrestrial Research, New Jersey Institute of Technology, Newark, NJ 07102-1982, USA \\ ${ }^{8}$ Space Research Laboratory, Department of Physics and Astronomy, University of Turku, Turku FI-20014, Finland \\ Received 2021 April 15; revised 2021 May 5; accepted 2021 May 7; published 2021 June 29
}

\begin{abstract}
During the 24th solar cycle, the Fermi Large Area Telescope (LAT) has observed a total of 27 solar flares possessing delayed $\gamma$-ray emission, including the exceptionally well-observed flare and coronal mass ejection (CME) on 2017 September 10. Based on the Fermi/LAT data, we plot, for the first time, maps of possible sources of the delayed $>100 \mathrm{MeV} \gamma$-ray emission of the 2017 September 10 event. The long-lasting $\gamma$-ray emission is localized under the CME core. The $\gamma$-ray spectrum exhibits intermittent changes in time, implying that more than one source of high-energy protons was formed during the flare-CME eruption. We find a good statistical correlation between the $\gamma$-ray fluences of the Fermi/LAT-observed delayed events and the products of corresponding CME speed and the square root of the soft X-ray flare magnitude. Data support the idea that both flares and CMEs jointly contribute to the production of subrelativistic and relativistic protons near the Sun.
\end{abstract}

Unified Astronomy Thesaurus concepts: Solar flares (1496); Solar energetic particles (1491); Solar gamma-ray emission (1497)

\section{Introduction}

Impulsive-gradual classification of solar energetic particle (SEP) events was formulated for electrons and ions with energies well below $100 \mathrm{MeV}$ (Cane et al. 1986; Cliver 1996, and references therein). It currently links impulsive SEP events to solar flares, while the gradual (long-duration) SEP events are ascribed to the CME shock acceleration in the solar wind (Reames 1999). However, in the context of extreme SEP events affecting the Earth's atmosphere and systems in space and on the ground, solar protons of much higher energies, $\gtrsim 300 \mathrm{MeV}$ (e.g., Cliver et al. 2020), are essential. Protons of such high energies, if they precipitate back to the Sun, can produce, via nuclear interactions, the $>100 \mathrm{MeV} \gamma$-rays detected by the Fermi Large Area Telescope (LAT; Atwood et al. 2009). In this paper, we focus on the high-energy protons interacting at the Sun and the interplanetary protons arriving at 1 au whose origins are presently not well known.

Historic $\gamma$-ray observations of the 1991 June flares on the Compton Gamma Ray Observatory and GAMMA-1 satellites led to a conclusion that during the $8 \mathrm{hr}$ duration postimpulsive phase of the June 11 flare there were at least three distinct episodes of high-energy proton acceleration at the Sun (Mandzhavidze et al. 1996). Observations of the 2000 July 14 solar flare were analyzed by Klein et al. (2001), indicating that after the flare's impulsive phase, the relativistic protons were accelerated in the course of the magnetic field reconfiguration at heights between 0.1 and $1 R_{\odot}$ above the

Original content from this work may be used under the terms of the Creative Commons Attribution 4.0 licence. Any further distribution of this work must maintain attribution to the author(s) and the title of the work, journal citation and DOI. photosphere, and not in the flaring active region or at the bow shock of the CME. Several episodes of high-energy particle acceleration in the corona within $\approx 1 R_{\odot}$ were revealed in the 2005 January 20 event (Masson et al. 2009). Data of historic long-duration $\gamma$-ray flares favored either acceleration of protons and ions for long periods of time by second-order Fermi acceleration in large coronal loops, or acceleration in largescale, CME-associated reconnection sheets (see the review by Ryan 2000 and references therein). An alternative hypothesis reads in application to the $\gamma$-ray flare and the ground level enhancement (GLE) event on 2017 September 10 that most of the high-energy protons, including even protons interacting during the flare's impulsive phase, were accelerated by the CME-driven shock outside the flaring active region (Kouloumvakos et al. 2020). In contrast, de Nolfo et al. (2019) argue that the back precipitation of shock-accelerated particles is unlikely to be the source of the long-duration $\gamma$-ray emission. Hutchinson et al. (2020) have studied the back-precipitation efficiency by means of test-particle simulations and conclude that the time-extended acceleration and large total precipitation fractions, as seen in the $\gamma$-ray observations, cannot be reconciled for a moving CME bow shock.

A knowledge of the $\gamma$-ray source size and location on the Sun would be crucial to distinguish them among different models. However, the point-spread function of the Fermi/LAT in the solar context is wide, about four solar radii at $1 \mathrm{GeV}$, so that even a global $\gamma$-ray source extending through the entire solar hemisphere may seem consistent with the LAT data. In this paper, we show that such an understanding is not correct for events like 2017 September 10, in which the $\gamma$-ray emission's centroid is located close to the solar limb. 


\section{High-energy Event Morphology and Associations}

The high-energy $\gamma$-ray flare on 2017 September 10 was observed by Fermi/LAT (Omodei et al. 2018; Ajello et al. 2021), and the high-energy proton event at 1 au was registered by the ground-based neutron monitor network (Mishev et al. 2018; Kocharov et al. 2020). The flare-CME eruption was well observed in different electromagnetic emissions, including in particular the microwave (MW) observations by the Expanded Owens Valley Solar Array (EOVSA; Gary et al. 2018), the EUV images by the Atmospheric Imaging Assembly (AIA; Lemen et al. 2012) on the Solar Dynamics Observatory (SDO), and the hard X-ray (HXR) observations by RHESSI (Lin et al. 2002).

Figures 1 and 2 give an overview of the event. Time profiles of high-energy $\gamma$-rays and interplanetary protons are shown in Figure 1. Data of the $\gamma$-ray registration with Fermi-LAT have been fitted similarly to Omodei et al. (2018), and data of the GLE registration with the neutron monitor network have been fitted using a procedure described by Mishev et al. (2018).

Based on spectral evolution of the $\gamma$-ray event, we divide it into four phases labeled in Figure 1(c). Phase 1 is identical to the flare's impulsive phase. The main acceleration of the CME and the MW burst began at 15:49 UT-15:50 UT (Cheng et al. 2018; Chen et al. 2021, their Figure 17 and Figure 1, respectively), so 15:50 UT is adopted as the reference time for our Figure 1. Simultaneously, a global EUV wave, connected with the CME-driven shock, was launched (Liu et al. 2018; Hu et al. 2019). At the peak time of the flare's impulsive phase, $\approx 16: 00$ UT, the EUV wave was already well outside the flaring active region, far from the flare loops in which the impulsive-phase MW emissions were produced.

Time profiles of the MW emissions observed by EOVSA are frequency dependent. For Figure 1(b), we have selected an $\sim 8 \mathrm{GHz}$ profile, because its peak time coincides with the peak time of the $>100 \mathrm{MeV} \gamma$-ray emission observed by Fermi/ LAT. EOVSA's multifrequency MW images indicate that the $\sim 8 \mathrm{GHz}$ emission concentrates at or near the bottom of the CME-trailing current sheet above the flare loops, in a magnetic bottle around the $Y$-type neutral point of magnetic field (the flare loops at a later instant of time are shown in Figure 2(a), and the main flare arcade 1 in (b); more MW images and analysis are given in Gary et al. 2018; Chen et al. 2020a, 2021).

During the impulsive phase, the high-energy $\gamma$-ray spectrum is hard, with cutoff energy $E_{\mathrm{c}}^{(1)} \approx 250 \mathrm{MeV}$ (Figure 1(d)), produced by $\gtrsim 300 \mathrm{MeV}$ protons with an energy spectrum as hard as $N\left(E_{p}\right) \sim E_{p}^{-3.2}$ interacting at the Sun. However, no energetic particles were injected at that time into the solar wind on the Earth-connected magnetic field lines. According to the SEP registration data at/near the Earth, the SEP emission at the roots of the interplanetary magnetic lines started at 16:05 UT (solar time +8 minutes; Figure 1 of Kocharov et al. 2020, hereafter KPL20), i.e., at the transition from $\gamma$-ray Phase 1 to Phase 2. At that time, the Earth was magnetically connected to the highly compressed, eastern flank of the CME-driven shock near the Sun (Figure 5 of KPL20).

All observational data fit well with the traditional understanding that, during the flare's impulsive phase, protons can be accelerated to high energies on closed magnetic field lines at the flare location. The 2017 September $10 \gamma$-ray flare was bright enough to do a time-resolved localization study. The main flare arcade is inside the deduced centroid of the

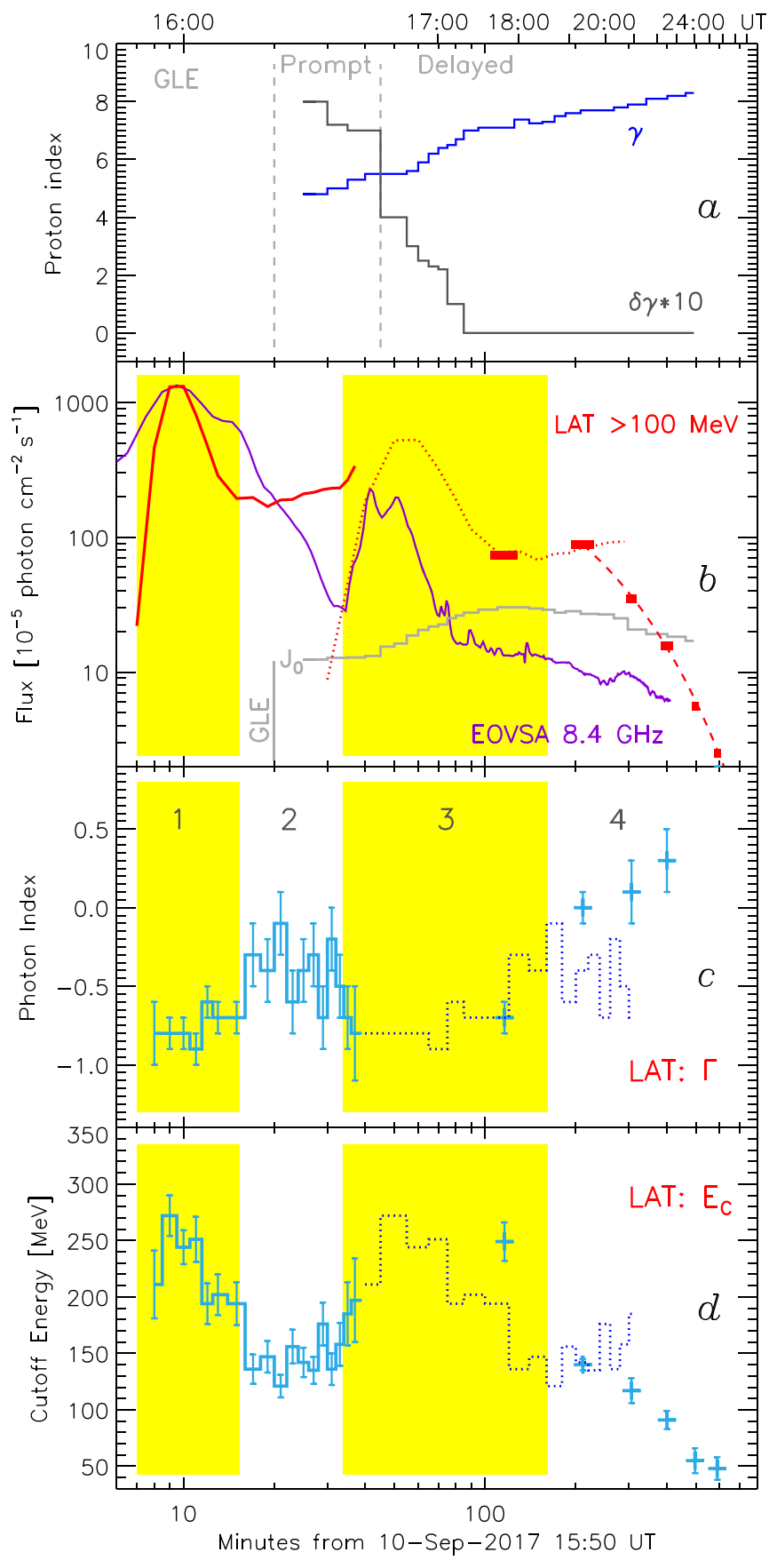

Figure 1. Two components of GLE (prompt and delayed) and four phases of the high-energy $\gamma$-ray emission (1-4) of the solar flare-CME eruption on 2017 September 10. (a) Time development of the GLE spectrum. The proton rigidity spectrum is of the form: $J_{\|}(P)=J_{0} P^{-\gamma+\delta \gamma(P-1 \mathrm{GV})}$, where rigidity $P$ is measured in $\mathrm{GV}, J_{\|}$is the intensity of protons arriving from the Sun along the distribution symmetry axis. (b) The high-energy $\gamma$-ray profile observed with Fermi/LAT (red solid line and blocks, with data gaps caused by the orbit limitations), compared with the normalized profile of MW emission observed with EOVSA, and the GLE intensity magnitude $J_{0}$ (in units of $0.5 \mathrm{~cm}^{-2} \mathrm{~s}^{-1}$ $\left.\mathrm{sr}^{-1} \mathrm{GV}^{-1}\right) .(c),(d)$ The photon spectral index, $\Gamma$, and cutoff energy, $E_{\mathrm{c}}$, of the best-fit $\gamma$-ray spectrum $d N_{\mathrm{ph}}(E) / d E=N_{0} E^{\Gamma} \exp \left(-E / E_{\mathrm{c}}\right)$. Dotted profiles in $(b)-(d)$ are for a possible interpolation through the data gap (explained in the text). The dashed red line in $(b)$ is an exponential fit to the time profile of the $\gamma$ ray decay.

$>100 \mathrm{MeV} \gamma$-ray emission at 15:57 UT-16:07 UT (Omodei et al. 2018; Ajello et al. 2021).

The GLE event on 2017 September 10 followed the general GLE pattern, which is that an enhancement starts with a prompt 


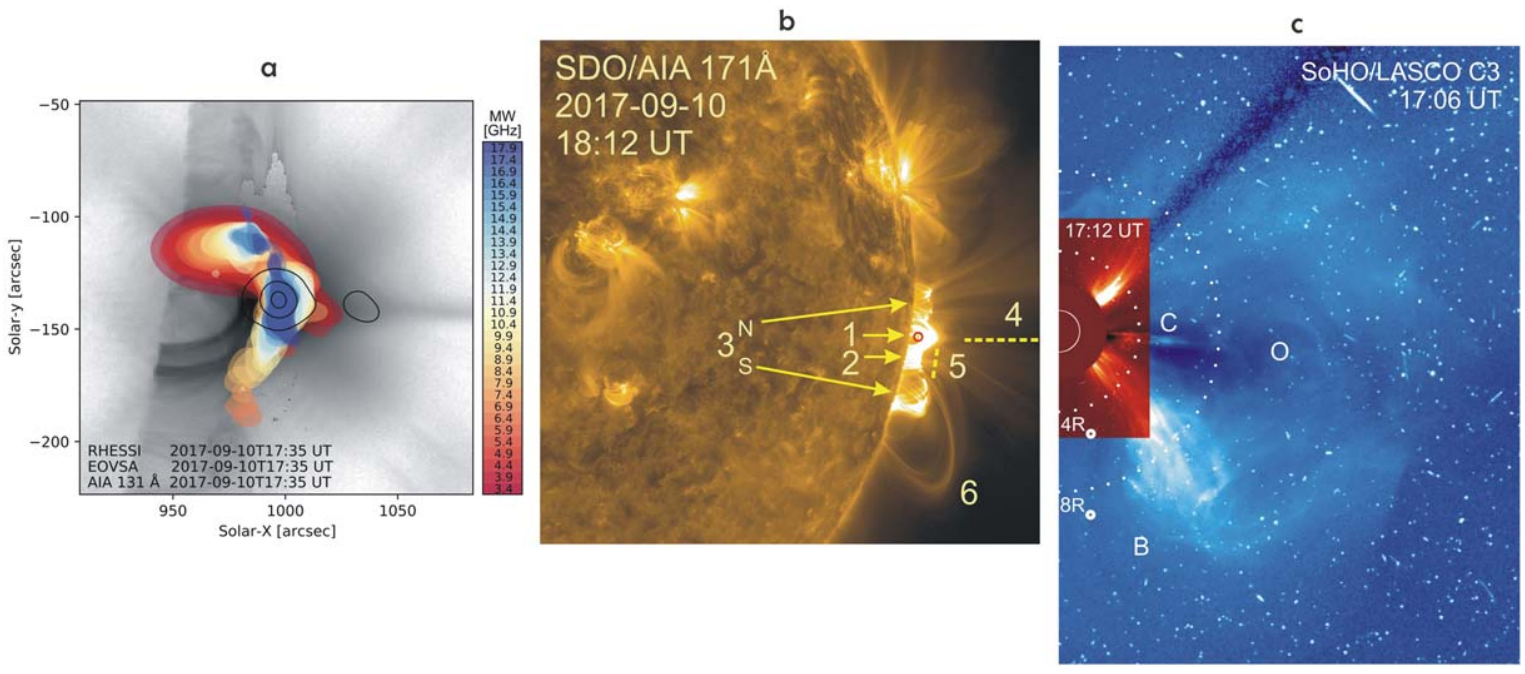

Figure 2. Flare-CME structures at around the peak time of the delayed $\gamma$-ray emission. (a) MW-emission frequency map by EOVSA (color coded similarly to Yu et al. 2020) and the 6-12 keV X-ray map by RHESSI (black contours at 10\%, 50\%, and 90\% of the maximum), superimposed on the SDO/AIA $131 \AA$ image (gray) at the end of Phase 3 of the $\gamma$-ray flare. (b) The AIA $171 \AA$ image at 18:12 UT. The main flare arcade (1) continuously rises up. The red circle specifies the 30-100 keV X-ray source location observed by RHESSI at 15:54 UT (the $\gamma$-ray Phase 1). The southern flare arcade (2) is seen in the soft X-ray images by GOES 15/Solar X-ray Imager already in Phase 1. In EUV images, its brightening expands to the south during Phase 2 and early Phase 3. In mid-Phase 3, the pair arcades (3) simultaneously brighten up to the north and the south of the main flare arcade. In Phase 4, the southern arcades (3S) exhibit numerous rapid evolutions (with no new brightening in soft X-rays). Also specified are the CME-trailing reconnection current sheet (4) with its southern extension (5; Chen et al. 2020b) and the high, rising loops (6) that disappear during Phase 4 at around 20:27 UT on 2017 September 10. (c) The Large Angle and Spectrometric Coronagraph Experiment (LASCO)/C3 image showing the CME core/magnetic flux rope (O), the bright blob (C) injected along the CME-trailing current sheet, and the southern CME loops (B). The blob leads a wider ejection seen in the 17:12 UT image by LASCO/C2 (red insert).

component whose rigidity spectrum steepens with rigidity in the GV range and continues with a delayed component possessing a power law rigidity spectrum (Figure 1(a)). Onset of the prompt component was observed in the beginning of Phase 2, while the delayed component started in the beginning of Phase 3 and continued in Phase 4 (panel (b)). The Phase $2 \gamma$ ray spectrum was markedly softer than the spectrum of Phase 1 , $E_{\mathrm{c}}^{(2)} \approx 150 \mathrm{MeV}$, so that the interacting protons of the early postimpulsive phase (Phase 2) were different from the protons of the impulsive phase (Phase 1).

After Fermi/LAT's nightfall at 16:29 UT, the next observation interval started at 17:33 UT, when a hard-spectrum $\gamma$-ray flux was observed (Figure 1), and hence the softspectrum flux of Phase 2 should decay between 16:30 UT and 17:30 UT. That was also a time interval when many radio bursts ended. Intense radio bursts in the $2-16 \mathrm{MHz}$ range, including the low-frequency type III emissions, were observed by STEREO A/WAVES to decay at 16:30 UT-16:50 UT (Figure 9 of KPL20). Corresponding radio bursts at 25-60 $\mathrm{MHz}$ continued through $\approx 16: 25$ UT (data from the San Vito Solar Observatory; https://ngdc.noaa.gov/stp/space-weather/ solar-data). In the 20-80 MHz frequency range, high-resolution data including images are available from the Low Frequency Array (LOFAR) for the time interval 15:50 UT-16:10 UT (Morosan et al. 2019). The LOFAR-observed radio emissions are associated with a lateral expansion of CME near the Sun, and include type II lanes and several herringbone structures, indicative of a quasi-perpendicular shock. In particular, a few minutes before the injection of the first SEPs to the Earthconnected magnetic field lines, the herringbone emissions were produced at the southern flank of the CME shock bubble (Figure 2 of Morosan et al. 2019). One more association for the $\gamma$-ray emission of Phase 2 is the observed signatures of vigorous magnetic reconnection at the top of the southern flare arcade, including plasma flows and oscillations (the arcade 2 in our Figure 2(b); Reeves et al. 2020).

Postimpulsive phase $\gamma$-ray emissions, including also $\pi^{0}$-decay $\gamma$-rays, were observed in the 1991 June 11 and June 15 flares to continue for up to 8 hours, and the observed $\gamma$-ray profiles were similar to the time profiles of MW emissions (Mandzhavidze et al. 1996; Kocharov et al. 1998, and references therein). During the Phase 3 time interval on 2017 September 10, the Fermi/LAT observations were possible only in the very beginning and at the end of the interval (Figure 1(b)), but the MW profiles are available and they could be used for an interpolation between the observed parts of the $\gamma$-ray profile. However, in an attempt to interpolate both the intensity and the spectral parameters, $\Gamma$ and $E_{\mathrm{c}}$, we have adopted a slightly different approach. The observed $\gamma$-ray profile of Phase $1+2$ was widened tenfold in both directions from 16:00 UT to mimic a slower evolution and then shifted for +50 minutes. The $\gamma$-ray flux has been also renormalized by factor of 0.4. The resulting profiles are shown with dashed lines in Figures 1(b)-(d). They provide a reasonable interpolation through the Fermi/LAT night.

Based on available images of the solar corona, we can see that the $\gamma$-ray Phase 3 starts with the launch of a narrow secondary ejection along the CME-trailing current sheet, the bright blob C in Figure 2(c), with a velocity in the LASCO field of view of $977 \pm 68 \mathrm{~km} \mathrm{~s}^{-1}$ (Lee et al. 2020, Blob-1 in Figure 1 therein) and the projected launch time 16:18 UT-16:22 UT at $0.1 R_{\odot}$. With the beginning of the coronameter observations at the Mauna Loa Solar Observatory (MLSO) at 17:11 UT, a sequence of intermittent antisunward moving blobs along the current sheet were seen in the MLSO field of view until 19:00 UT, with final velocities up to $1200 \mathrm{~km} \mathrm{~s}^{-1}$ (Cheng et al. 2018). During the flare's multi-hour-long decay, the recurring bidirectional outflows were seen in EUV to originate in the plasma sheet from discrete sites, identified as magnetic 
reconnection sites (X-type neutral points) residing below $0.26 R_{\odot}$, above the top of the main flare arcade ( $\mathrm{Yu}$ et al. 2020). The EUV observations revealed downward contractions of collapsing loops that impinge the top of the main flare arcade, from the beginning of the $\gamma$-ray flare's Phase 3 onwards (Figure 7 of Hayes et al. 2019). The sunward outflows caused quasiperiodic pulsations in EUV, soft X-ray, HXR, and MW emissions (Figure 2 of $\mathrm{Yu}$ et al. 2020, seen also in the MW profile shown in our Figure 1(b)). Those pulsations weakened after the end of the $\gamma$-ray Phase 3 .

MW and HXR emissions of Phase 3 are produced around the main flare arcade. They include a thermal 10-20 MK source at the loop top and a nonthermal MW source in the north loop-leg region, plus an extension of the MW source over the southern flare arcade (Figure 2(a)). New EUV brightenings extend to a wider range of loops on both sides of the CME-trailing current sheet (arcades $3 \mathrm{~N}$ and $3 \mathrm{~S}$ in Figure 2(b)).

The delayed component of the GLE observed by neutron monitors on 2017 September 10 began to grow in the beginning of Phase 3. Interplanetary transport modeling of the GLE-producing protons revealed that the delayed component began with the proton injection at the root of the Earthconnected magnetic field tube at 16:28 UT-16:33 UT (solar time +8 minutes; source $J_{2}$ in Figure 8 (a) of KPL20), but the peak intensity was achieved with the proton arrival across the interplanetary magnetic field lines from solar locations around the flaring active region near the west limb (source $G$ ). It was also found that the maximum of the source $G$ injection coincided in time with the maximum of the Phase 4 softspectrum $\gamma$-ray emission. The soft-spectrum component might exist already in Phase 3, but it could not be seen before the hard-spectrum $\gamma$-ray emission of Phase 3 had decayed.

In Phase 4, the $>100 \mathrm{MeV} \gamma$-ray flux rose until $\sim 19: 20 \mathrm{UT}$ and then exponentially decayed with an e-folding time of 1.8 hour (shown with the dotted line in Figure 1(b)). The delayed component of the GLE also decayed but with a longer decay time. In the late phase of the flare, the main flare arcade continued to rise (Figure 5 of French et al. 2020) and a number of fast evolutions were observed in the southern loop system (Figure 2(b)), while MW and HXR emissions declined. A prominent association is the low-frequency type II radio burst observed by STEREO A/WAVES at 16:50 UT-19:40 UT on 2017 September 10 between $0.3 \mathrm{MHz}$ and $1.6 \mathrm{MHz}$ (Figure 9 of KPL20).

\section{Gamma-ray Source Localization}

In order to produce high-energy $\gamma$-ray emission, $>300 \mathrm{MeV}$ protons must be accelerated in some incompletely known parts of solar corona, possibly including the solar wind, and subsequently precipitate into the chromosphere. Mandzhavidze $\&$ Ramaty (1992) investigated the $\gamma$-ray production resulting from pion decay taking account, in particular, of the transport of the $\gamma$-rays in the solar atmosphere. As the pions are produced by high-energy protons quite deep into the solar atmosphere, Compton scattering and electron-positron pair production introduce significant modifications to the flux and spectrum of the escaping $\gamma$-rays if the viewing direction is close to tangential to the solar sphere, $\cos \theta \lesssim 0.1$, where $\theta$ is the angle between an upward-directed solar radius vector and the direction of observation (the direction perpendicular to the plane $X-Y$ in our Figure 3). At $\cos \theta>0.2$, the emission source is optically thin, and hence its surface brightness is proportional to the source's depths along the line of view: $w \propto 1 / \cos \theta$.

For a simplistic calculation, we adopt the surface brightness as a function of the plane-of-sky coordinates $X$ and $Y$ (Figure 3) in the $w(X, Y)=b / \sqrt{\cos ^{2} \theta+\varepsilon^{2}} \equiv b / \sqrt{1-X^{2}-Y^{2}+\varepsilon^{2}}$, where $b$ is a constant proportional to the density of the vertical precipitation rate of high-energy protons; model parameter $\varepsilon$ accounts for the emission attenuation near the limb $(\varepsilon=0.1)$. All the coordinates are in solar radius units. Integration of the surface brightness over the visible source area $(S)$ yields the source's total brightness $w_{S}=\iint_{S} w(X, Y) d X d Y$. The center of brightness coordinates of the model source are:

$$
\begin{aligned}
X_{\mathrm{cb}} & =w_{S}^{-1} \iint_{S} X w(X, Y) d X d Y, \\
Y_{\mathrm{cb}} & =w_{S}^{-1} \iint_{S} Y w(X, Y) d X d Y .
\end{aligned}
$$

We consider a $\gamma$-ray source that is bounded by a circle on the solar sphere. The radius of the source-bounding circle is measured in units of the heliocentric angle, $\alpha$, which is the generator angle for the right circular cone with its apex at the solar center and base on the source's circle. The source's geometric center is at the intersection of the cone's axis with the solar sphere, while the brightness center may be at a different location depending on the viewing direction. The brightness center location of the model source will be adjusted to a location assessed from the Fermi-LAT data.

As examples, in Figure 3(a) we plot a few different sources that have, for a near-Earth observer, the same brightness center, which is shown with a diamond (overlaid on solar $131 \AA$ images by SDO/AIA). All sources have the brightness center fixed at the easternmost point of the LAT centroid at 19:03 UT-19:39 UT (the centroid is defined as the 95\% confidence-level localization of the emission center; Omodei et al. 2018). In panel (b), the same brightness center and the same sources are shown in projection onto the plane rotated $50^{\circ}$ west. Also shown are the geometric centers of the sources and their (nominal) parts not visible by Fermi-LAT (thin contours).

In Figure 3(b), we additionally show the proton injection sector for the interplanetary transport modeling of the event delayed phase (source $G$ in Figures 7 and 8 of KPL20), which is consistent with the location of the delayed $\gamma$-ray source produced by protons precipitating to the Sun. This supports the idea that both proton populations originate from a common source situated within $30^{\circ}$ from the west limb. Note that the model proton flux near the Earth is not sensitive to the adopted west boundary of the injection sector $G$, so it could be extended farther to the west, while the east boundary location is essential.

With an increase of the source radius $\alpha$ at a given position of the brightness center, the geometric center of the source moves westward, eventually crossing the west limb. The source's eastern extension reaches its maximum value at $\alpha \approx 15^{\circ}$. The largest visible area is achieved at $\alpha \approx 25^{\circ}$. The visible source could not occupy more than $2.7 \%$ of the total area of the solar disk. This is slightly less than the area of the LAT-measured centroid.

In panel (c), the bounded area includes circular sources of all possible sizes and all possible brightness center locations that are consistent with the Fermi-LAT centroid, still with a limitation that the source circle center is not farther than $20^{\circ}$ 

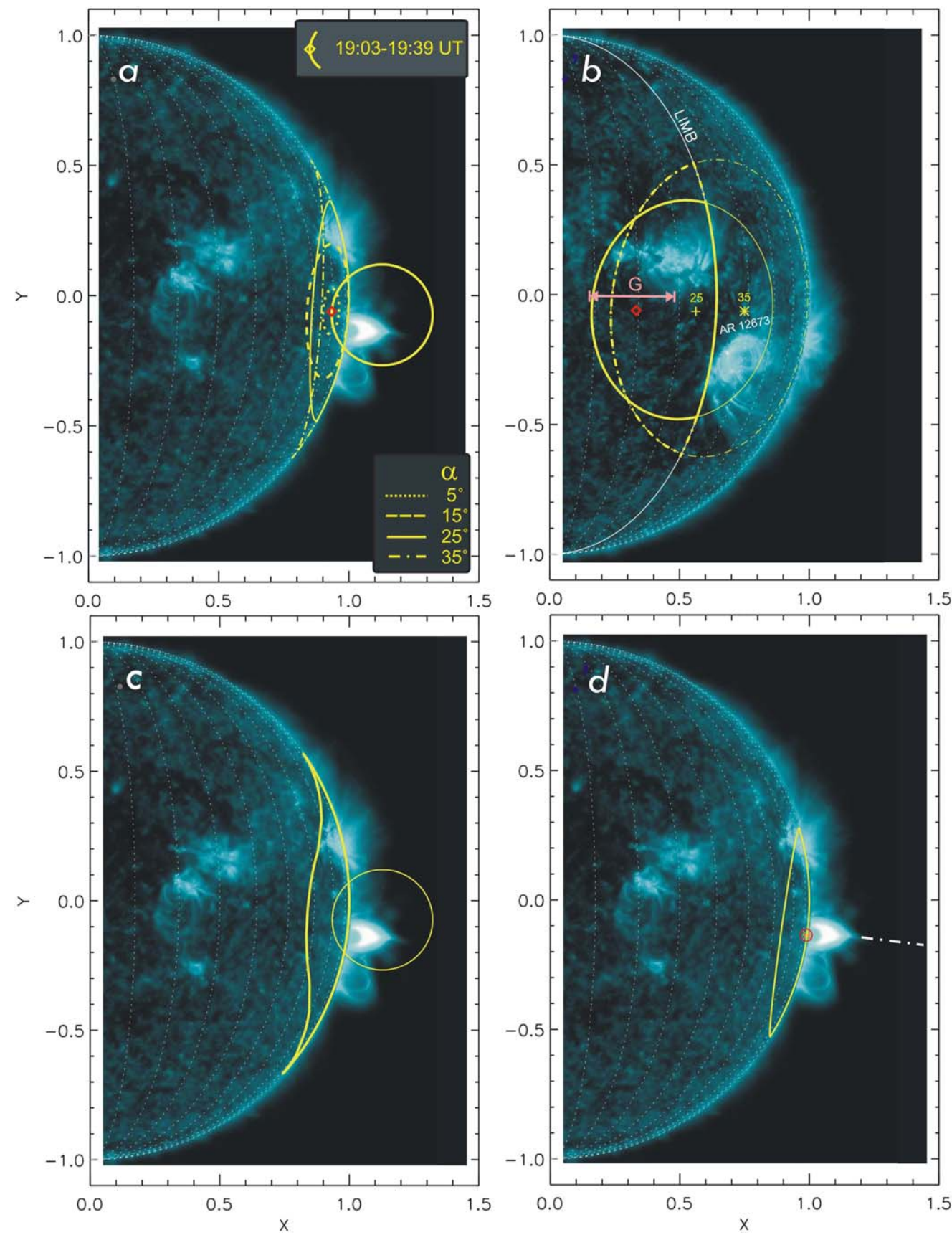

Figure 3. Maps of possible high-energy $\gamma$-ray sources consistent with the emission's centroid observed with Fermi-LAT (yellow circle) in the late phase of the 2017 September 10 event. The background image is the SDO/AIA $131 \AA$ image at 22:42 UT on September 10 (except panel $(b)$ ). $(a)$ The case of the $\gamma$-ray brightness center being fixed at the east edge of the centroid (red diamond). (b) A side view of the same case, from $50^{\circ}$ west of the Earth, overlaid on an earlier SDO/AIA image taken on 2017 September 7 at 04:13 UT (due to inclination of the solar rotation axis, AR 12673 is at somewhat smaller values of $Y$ compared to September 10); the plus sign and asterisk indicate the geometric center location for sources of two different sizes, $\alpha=25^{\circ}$ and $\alpha=35^{\circ}$, respectively; additionally shown is the injection sector $G$ adopted in KPL20 for the interplanetary transport modeling of the extended phase of the GLE event. (c) Locus of all possible $\gamma$-ray sources on the visible hemisphere of the Sun corresponding to the Fermi-LAT centroid. $(d)$ The largest possible $\left(\alpha=24^{\circ}\right)$ source centered at the flare site (red circle) and the CME current sheet (dashed-dotted line).

beyond the west limb. The contour shown in panel (c) outlines the area of all possible sources, i.e., no one source extends outside this contour, and each and every point inside the contour belongs to some source consistent with the observed centroid. In this sense, the contour maps the circle of the FermiLAT centroid onto the solar disk. Alternatively, the geometric center of a source can be fixed at the flare location in AR 12673, while the source size is varied. The source shown in panel (d) is the largest possible flare-centered source whose brightness center is still located within the Fermi-LAT centroid.

\section{Statistical Consideration}

The source of the delayed $\gamma$-ray emission on 2017 September 10 did not extend farther than $35^{\circ}$ east of the west limb, while the flaring active region was near the west limb (Figure 3(a)). Fermi/LAT observed three other events with $\gamma$-ray emission from flares originating from active regions located up to $36^{\circ}$ 


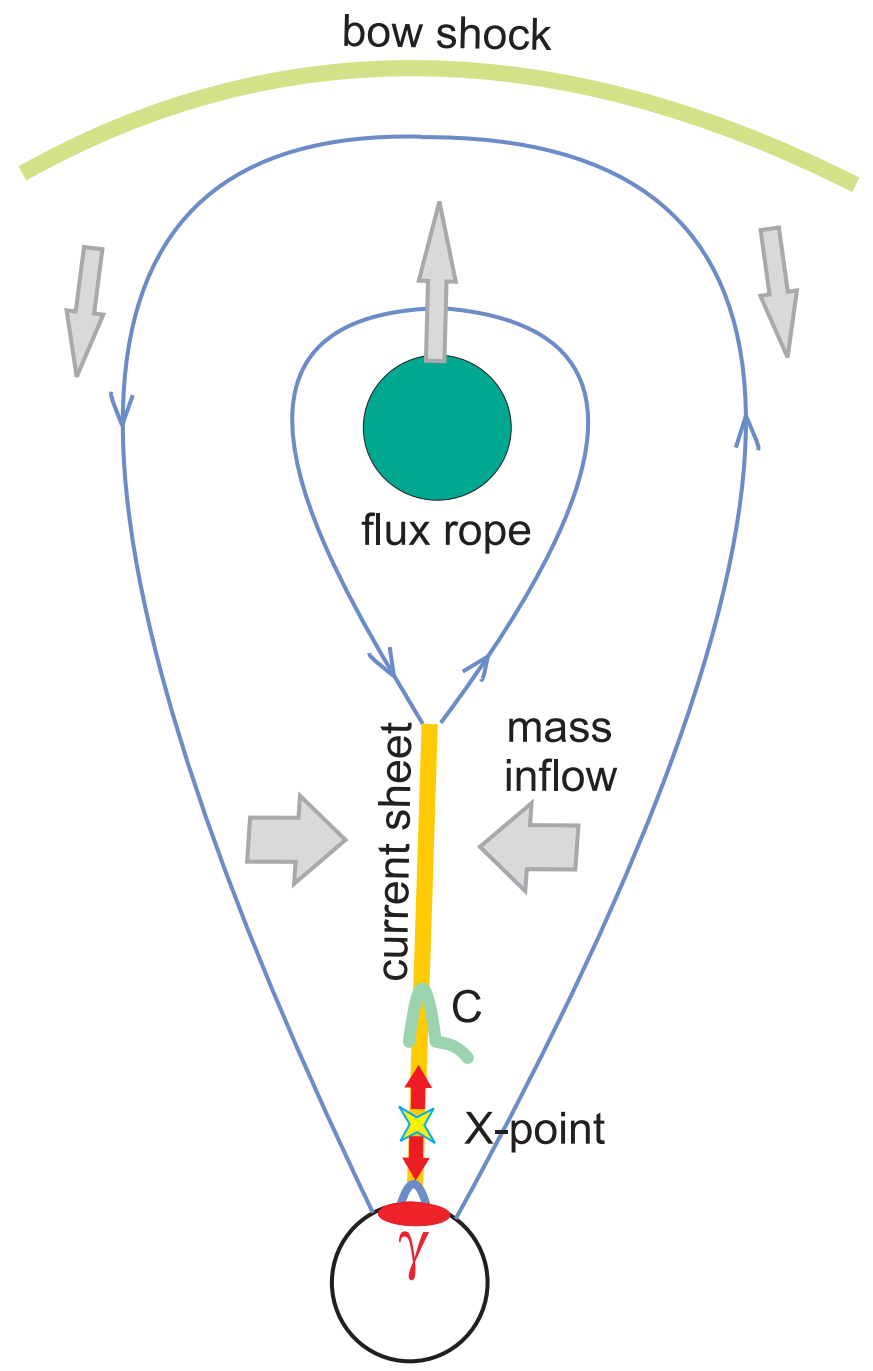

Figure 4. Schematic showing the locus of all possible high-energy $\gamma$-ray sources, situated beneath the CME-trailing current sheet with a reconnection point $(\mathrm{X})$, from where the bidirectional outflows originate, and the secondary ejection (C), like one seen in Figure 2(c).

behind the limb of the visible solar disk (Ajello et al. 2021). For this reason, it is most likely that the actual size of the $\gamma$-ray source on 2017 September 10 was not very far from its upper estimate obtained in Section 3. As a compromise, we adopt the source's half width $\alpha \sim 20^{\circ}$. The possible source of the delayed $\gamma$-ray emission on 2017 September 10 is sketched in Figure 4. A straightforward explanation for its location around the flaring active region under the $\mathrm{CME}$ core may be that $\mathrm{CME}$ only reaccelerates the originally flare-accelerated protons.

Fermi/LAT observed a total of $45 \gamma$-ray flares with emission in the energy band $30 \mathrm{MeV}-10 \mathrm{GeV}$ from 2010-2018. In a large number of the flares the $\gamma$-ray emission was detected beyond the end of the HXR burst. That general category is referred to as delayed emission if the $\gamma$-ray emission was seen in at least one Fermi orbit that does not include the flare's impulsive phase (the events are listed in Table 1 of Ajello et al. 2021). For 26 delayed events with flare-CME parameters given in Table 4 of Ajello et al. (2021), we study correlations between the $>100 \mathrm{MeV} \gamma$-ray fluence, $F$, and parameters of the associated flare-CME eruption.

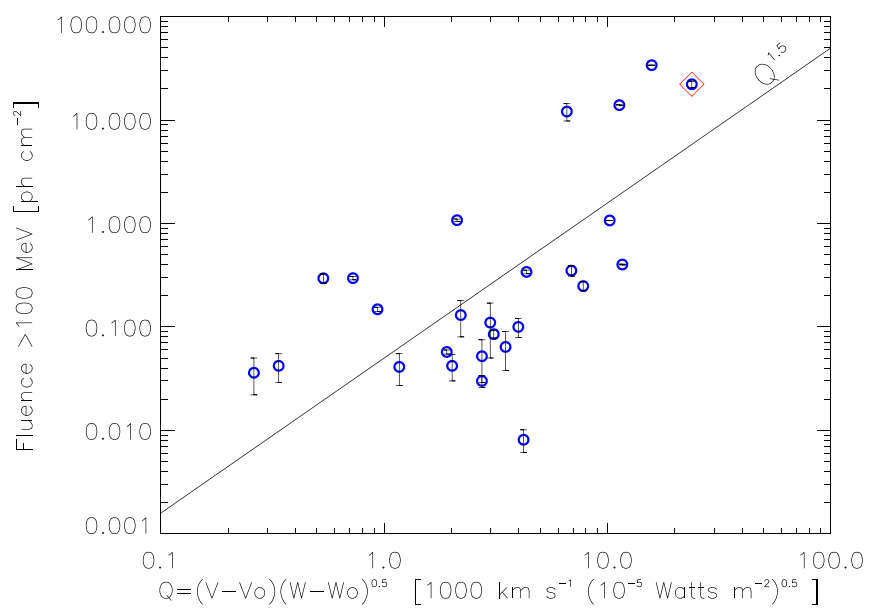

Figure 5. Scatter plot of the $>100 \mathrm{MeV} \gamma$-ray fluence of the delayed-type $\gamma$-ray flares vs. the product of associated CME velocity and square root of the X-ray flare magnitude. Adopted threshold values are: the Solar and Heliospheric Observatory/LASCO CME speed $V_{\mathrm{o}}=500 \mathrm{~km} \mathrm{~s}^{-1}$ and the GOES flare class M2.0 $\left(W_{\mathrm{o}}=2 \times 10^{-5} \mathrm{~W} \mathrm{~m}^{-2}\right)$. The red diamond specifies the 2017 September 10 eruption.

Figure 5 shows a scatter plot of the $>100 \mathrm{MeV} \gamma$-ray fluence of delayed events versus the combined flare-CME parameter

$$
Q=\left(V-V_{\mathrm{o}}\right)\left(W-W_{\mathrm{o}}\right)^{0.5},
$$

where $W$ is the soft X-ray flare magnitude, $V$ is the LASCO CME speed, and $V_{\mathrm{o}}$ and $W_{\mathrm{o}}$ are empirical threshold values, which are $V_{\mathrm{o}}=500 \mathrm{~km} \mathrm{~s}^{-1}$ and $W_{\mathrm{o}}=2 \times 10^{-5} \mathrm{~W} \mathrm{~m}^{-2}$. We find a good correlation between the flare-CME parameter $Q$ and the $\gamma$-ray fluence of delayed events, with the correlation coefficient $r(Q, F)=0.77_{-0.10}^{+0.07}$. It is higher than the correlation coefficient between the $\gamma$-ray fluence and the CME speed, $r(V, F)=0.60_{-0.15}^{+0.11}$, or the correlation coefficient between the fluence and the soft X-ray flare magnitude, $r(W, F)=0.61_{-0.15}^{+0.11}$. This supports the idea that the delayed high-energy $\gamma$-ray emission is the result of flare-CME synergy.

Trottet et al. (2015) performed in-depth statistical analysis of relationships between the peak intensities of interplanetary deka-MeV protons and near-relativistic electrons, on the one hand, and parameters of the solar flare-CME eruptions, on the other hand. The partial correlation analysis indicated that the only significant quantities of the eruptions were the CME speed and the soft X-ray fluence, suggesting that both the solar flare and the CME shock contribute to the SEP production in large events. In future, we plan to apply a similar in-depth analysis to the $\gamma$-ray emissions observed by Fermi/LAT.

\section{Discussion}

Acceleration of high-energy protons by collisions against irregularities of the magnetic field is known as Fermi-type acceleration (Fermi 1949). Compressive and turbulent plasma flows associated with the solar flare-CME eruption provide a number of opportunities for Fermi acceleration, either of the first-order (regular) acceleration in compressive flows or of the second-order (stochastic) acceleration in turbulence of various kinds, or both.

Proton acceleration to (sub)relativistic energies begins in the impulsive phase of the 2017 September 10 flare (Phase 1, Figure 1). A "secondary flare" pulse is seen in the MW profile of Phase 3. Particle acceleration in the flare's impulsive phase 
proceeds in the environment where the newly reconnected magnetic field lines emanating from the CME-trailing current sheet interact with the underlying flare arcades. Particles can be accelerated there in collapsing magnetic traps (Somov \& Kosugi 1997) or by Fermi-type acceleration in the "termination" shocks formed by reconnection outflows of the CMEtrailing current sheet (Masuda et al. 1994; Chen et al. 2015; Kong et al. 2019). The flare environment is rich in turbulence, waves, and a fractal electric field, which have also been suggested as possible particle acceleration mechanisms (see the review by Zharkova et al. 2011, and references therein). Protons could be accelerated by the turbulence both on closed magnetic field lines of flare loops and in the overlying magnetic field. However, in case of the 2017 September 10 event, no energetic particles were observed to escape directly from the flare site to the Earth-connected magnetic field lines of the solar wind.

The $\gamma$-ray Phase 2 starts with a lateral expansion of the CME near the Sun, with radio signatures of highly oblique shocks at heliocentric distances $\sim 2 R_{\odot}$ (Morosan et al. 2019). Such shocks can efficiently accelerate both the interacting protons producing the pion decay $\gamma$-ray emissions at the Sun and the interplanetary protons responsible for the prompt component of the GLE at the Earth. Several relevant acceleration models were considered by Kocharov et al. (2011, 2012), Kong et al. (2017), and Kouloumvakos et al. (2020).

The GLE-producing proton event can be modeled with two sources-one source at the root of the Earth-connected interplanetary magnetic field line and another source situated within $30^{\circ}$ from the solar western limb (KPL20; sector $G$ of our present Figure 3(b)). The maximum phase of the $G$ injection of interplanetary protons coincides with the maximum phase of the delayed $>100 \mathrm{MeV} \gamma$-ray emission and with the end of the low-frequency type II radio burst at $\sim 19: 30$ UT observed by STEREO A/WAVES. For this reason, a CME-driven shock may be considered as the main accelerator for both the delayed component of interacting protons and the delayed component of the GLE. A model of this kind was proposed by Kocharov et al. (2015).

A physics-based model of the shock acceleration for the interacting protons producing $\gamma$-rays at the Sun runs into the problem of the proton precipitation back to the solar chromosphere, if the shock operates in the solar wind far from the Sun. The back precipitation of the shock-accelerated protons is not a "natural" property of a diffusive shock acceleration, because a strong particle scattering exists both upstream and downstream of the shock for a fast enough acceleration, and the required scattering is so strong that the downstream particle transport is dominated by advection in the direction of CME propagation, and hence the accelerated particles do not precipitate back to the Sun. In order to overcome this difficulty, one may assume that the turbulent region downstream of the shock is of a fixed depth and comoving with the shock (Vainio et al. 2000). Alternatively, Kocharov et al. (2015) propose that the highly turbulent magnetic tube (a "core tube" connected to the flaring active region) is surrounded by less turbulent magnetic tubes (quiet "halo tubes"), so that the high-energy protons are accelerated by the shock mainly in the highly turbulent core tube and then escape via cross-field diffusion to the halo tubes, through which they can either precipitate back to the Sun or escape to $1 \mathrm{au}$. Another process that can enhance the flux of high-energy protons precipitating back to the Sun is stochastic acceleration in the shock downstream region, which is slower than acceleration in the shock front but operates on a much longer timescale, and hence should not be neglected.

In the case considered by Kocharov et al. (2015), the shock acceleration was operating during the shock transit from the heliocentric distance of $1.6-6.4 R_{\odot}$, and the resulting ratio of the back-precipitating $>300 \mathrm{MeV}$ proton number to the upstream-escaping proton number was $N_{\downarrow} / N_{\uparrow} \approx 0.02$. During the $\gamma$-ray Phases 3 and 4, on 2017 September 10 between 16:30 UT and 19:00 UT, the LASCO CME expands from $12 R_{\odot}$ to $53 R_{\odot}$. It is $\approx 8$ times the CME height range in the modeling by Kocharov et al. (2015). Focused diffusion of protons along the interplanetary magnetic field tubes is formally equivalent to the radial diffusion with a renormalized diffusion coefficient (Kunow et al. 1991), and hence the probability of back precipitation decreases with distance from the Sun, $r$, approximately as $r^{-2}$, still neglecting a possible radial dependence of the diffusion coefficient. If so, the theoretical ratio $N_{\downarrow} / N_{\uparrow}$ for the 2017 September 10 CME bow shock can be estimated as $\approx 0.0003$. In contrast, the estimate based on the observational data for the delayed component $(G)$ is $N_{\downarrow} / N_{\uparrow}=0.002-0.01$ (KPL20). However, the location of the type II emission observed by STEREO A/WAVES is not known. If the type II was located at $(0.17-0.39)$ times the LASCO CME height (for the frame shown in Figure 2(c), it is $3.7 R_{\odot}-8.4 R_{\odot}$ ), the theoretical interacting-proton number could be consistent with the observational estimate. In such a case, the proton acceleration site may be located either near and downstream of the ejection $\mathrm{C}$ around the CME-trailing current sheet, or around the southern CME loops B (Figure 2(c)).

Particles can be accelerated also in plasma compression at the CME-trailing current sheet, in a head-on collision of two inflows sketched in Figure 4 (e.g., Drury 2012). Similar to particle acceleration in shock waves, the acceleration in the reconnection layer is a mixture of first- and second-order Fermi-type processes ( $\mathrm{Li}$ et al. 2018).

Compared to most solar particle events, GLEs and prolonged $>100 \mathrm{MeV} \gamma$-ray events are extreme, in terms of the (sub) relativistic proton number, and associated with major but not outstanding flares and CMEs, so a scenario of such events should include a synergy of flares and CMEs. There were spectroscopic signatures found in the $\gamma$-ray data that stochastic acceleration of interacting ions continues during the decay phase of a $\gamma$-ray flare (Ramaty et al. 2000). On a longer timescale, the ions may be further accelerated in other parts of the eruption.

Using the solar flare $\gamma$-ray spectroscopy and the interplanetary particle detection in the $\mathrm{MeV}$-to-deka-MeV energy range, Murphy et al. (1991) concluded that the interacting particle composition is different from the composition of particles observed in large proton events in space (gradual SEP events); rather, it resembles the composition observed in the impulsive SEP events which are rich in helium and heavy ions. Because of such a difference in the ion abundances between the interacting particles and interplanetary particles, those ion populations are ascribed to two different sources-the flare at the Sun and the CME shock in the solar wind. However, observations in the hecto-MeV-to-GeV range by the Payload for Matter-Antimatter Exploration and Light Nuclei Astrophysics in space (PAMELA; Adriani et al. 2011) indicated that both components of the 2006 December 13 GLE-the prompt component observed before $\approx 03: 50$ UT on 2006 December 13 


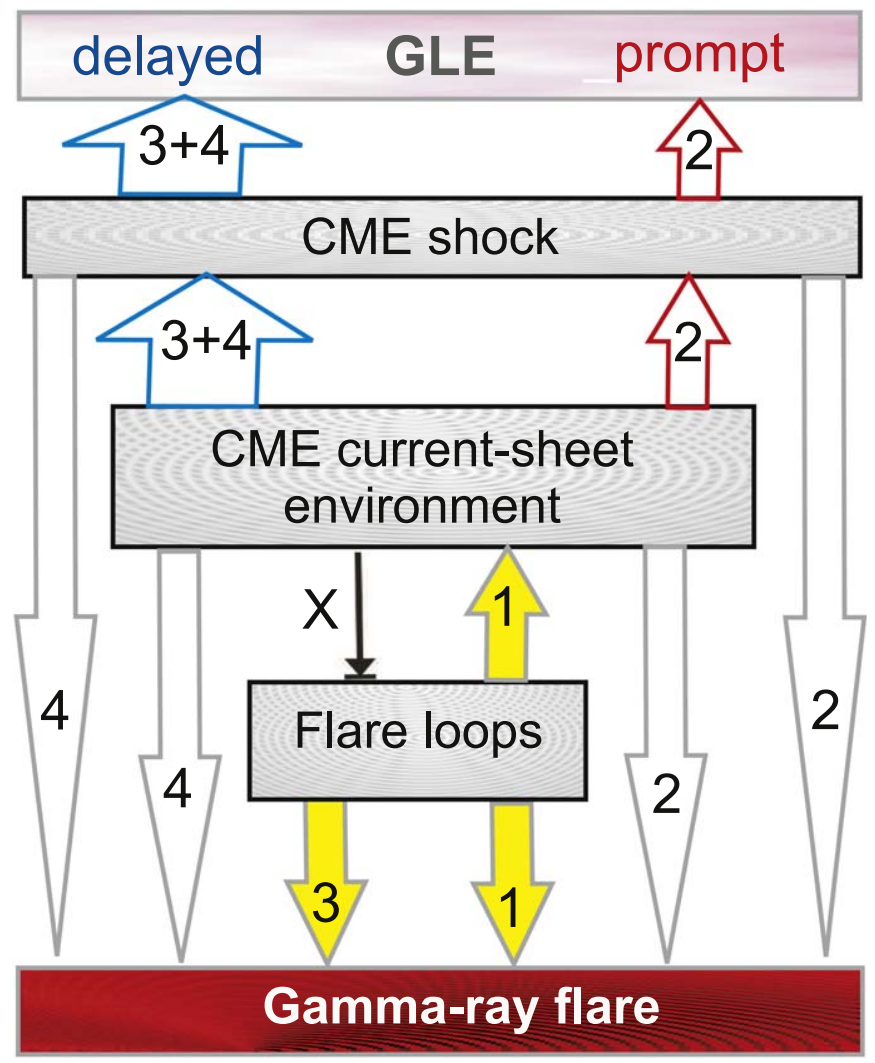

Figure 6. Schematic showing the possible scenario and locations of highenergy proton acceleration and reacceleration during the flare-CME eruption on 2017 September 10. Connection arrows illustrate the particle transfer episodes, labeled according to the $\gamma$-ray phase numbers defined in Figure 1. Arrow $\mathrm{X}$ symbolizes reconnection outflows affecting the flare arcade during Phase 3.

and the delayed component observed after that time-were rich in helium, similar to impulsive events of the flare origin. This supports the idea that the highest possible energies of solar particles are achieved when a CME reaccelerates the heliumrich composition of the flare origin.

The 2017 September 10 energetic ion event in the 7.3-25 $\mathrm{MeV} \mathrm{nucl}^{-1}$ energy range starts with a time interval of impulsive composition, $\mathrm{He} / p \approx 0.1,16: 10$ UT-17:10 UT, which coincides with arrival time of the GLE's prompt component (Figure 1(f) of KPL20). After that, the $\mathrm{He} / p$ abundance ratio decreases to $\mathrm{He} / p \sim 0.02$, which is (almost) the gradual composition expected in large SEP events. Spectroscopic observations of the plasma of the CME-trailing current sheet indicate that the current sheet is formed from the surrounding coronal plasma, not from the helium-rich plasma of ejection itself (Warren et al. 2018), so the MeV-to-deka$\mathrm{MeV}$ SEPs of a gradual composition could be freshly accelerated from the plasma around the CME-trailing current sheet, or from the solar wind plasma.

A possible scenario of the 2017 September 10 event is illustrated with Figure 6. Gamma-ray emission of Phase 1 is produced by the hard-spectrum protons accelerated on closed magnetic field lines around the flare loops. Simultaneously, some protons could populate the overlying volume around the CME-trailing current sheet. In Phase 2, those protons can be reaccelerated in the highly oblique shock driven by the lateral expansion of the CME near the Sun, a fraction of which precipitates to the chromosphere to produce the Phase $2 \gamma$-ray emission, while others escape to the solar wind as the GLE's prompt component. Hard-spectrum protons are accelerated for the second time in the "secondary flare" of Phase 3. In the same phase, a soft-spectrum proton population from the current-sheet environment gives rise to the GLE's delayed component. However, the main reacceleration phase of both the highenergy protons producing the delayed component of the GLE and the protons producing the high-energy $\gamma$-ray emission occurs at the beginning of Phase 4. The GLE's prompt component is released at the lateral expansion of the $\mathrm{CME}$ near the Sun, while the GLE's delayed component arrives though the CME bow shock from the magnetic trap below the CME core, around the CME-trailing reconnection current sheet.

\section{Conclusion}

We have analyzed the interplanetary SEP-GLE event and the $>100 \mathrm{MeV} \gamma$-ray burst of the major flare-CME eruption on 2017 September 10. The deduced location of the delayed $\gamma$-ray source is within $\approx 20^{\circ}$ of the heliocentric angle around the flare site, not what was expected in the CME-bow shock backprecipitation model by Kouloumvakos et al. (2020). We can resolve several components of high-energy ions. In the $\mathrm{MeV}$ to-deka-MeV energy range, the SEP event comprises two components - the event starts with emission of the helium-rich ion composition, followed by a gradual, helium-poor component. The GLE event comprises the prompt component and the delayed component that differ in their energy spectra and in their solar locations. The high-energy $\gamma$-ray data suggest four episodes of the high-energy proton acceleration at/near the Sun, two of which are located around the flare loops, while two others are situated in a wider region around the CME-trailing current sheet. Multiwavelength observations of the flare-CME eruption reveal multiple locations where the energetic protons could be produced by the first-order and second-order Fermitype acceleration. While production of energetic protons is a universal property of major solar eruptions, the available highresolution broadband data suggest that the proton acceleration model cannot be universally simple.

This research is supported by the Academy of Finland at University of Oulu (projects 330064 QUASARE and 321882 ESPERA). D.G. and S.U. acknowledge support from NSF grant AST-1910354 and NASA grant 80NSSC18K1128 to New Jersey Institute of Technology.

\section{ORCID iDs}

Leon Kocharov (iD https://orcid.org/0000-0002-2077-5618

Nicola Omodei (ib https://orcid.org/0000-0002-5448-7577

Melissa Pesce-Rollins (iD https://orcid.org/0000-00031790-8018

Francesco Longo (iD https://orcid.org/0000-0003-2501-2270

Sijie Yu (i) https://orcid.org/0000-0003-2872-2614

Dale E. Gary (iD https://orcid.org/0000-0003-2520-8396

Rami Vainio (iD https://orcid.org/0000-0002-3298-2067

Ilya Usoskin (iD https://orcid.org/0000-0001-8227-9081

\section{References}

Adriani, O., Barbadino, G. C., Bazilevskaya, G. A., et al. 2011, ApJ, 742, 102 Ajello, M., Baldini, L., Bastieri, D., et al. 2021, ApJS, 252, 13

Atwood, W. B., Abdo, A. A., Ackermann, M., et al. 2009, ApJ, 697, 1071 
Cane, H. V., McGuire, R. E., \& von Rosenvinge, T. T. 1986, ApJ, 301, 448 Chen, B., Bastian, T. S., Shen, C., et al. 2015, Sci, 350, 1238

Chen, B., Battaglia, M., Krucker, S., Reeves, K. K., \& Glesener, L. 2021 ApJL, 908, L55

Chen, B., Shen, C., Gary, D. E., et al. 2020a, NatAs, 4, 1140

Chen, B., Yu, S., Reeves, K. K., \& Gary, D. E. 2020b, ApJL, 895, L50

Cheng, X., Li, Y., Wan, L. F., et al. 2018, ApJ, 866, 64

Cliver, E. D. 1996, in AIP Conf. Proc. 374, High Energy Solar Physics, ed. R. Ramaty, N. Mandzhavidze, \& X.-M. Hua (Melville, NY: AIP), 45

Cliver, E. D., Hayakawa, H., Love, J. J., \& Neidig, D. F. 2020, ApJ, 903, 41

de Nolfo, G. A., Bruno, A., Ryan, J. M., et al. 2019, ApJ, 879, 90

Drury, L. O'C 2012, MNRAS, 422, 2474

Fermi, E. 1949, PhRv, 75, 1169

French, R. J., Matthews, S. A., van Driel-Gesztelyi, L., et al. 2020, ApJ, 900, 192

Gary, D. E., Chen, B., Dennis, B., et al. 2018, ApJ, 863, 83

Hayes, L. A., Gallagher, P. T., Dennis, B. R., et al. 2019, ApJ, 875, 33

Hu, H., Liu, Y. D., Zhu, B., et al. 2019, ApJ, 878, 106

Hutchinson, A., Dalla, S., Laitinen, T., et al. 2020, arXiv:2012.05146

Klein, K.-L., Trottet, G., Lantos, P., \& Delaboudinière, J.-P. 2001, A\&A, 373, 1082

Kocharov, L., Cho, K.-S., \& Valtonen, E. 2011, ApJ, 735, 4

Kocharov, L., Debrunner, H., Kovaltsov, G., et al. 1998, A\&A, 340, 257

Kocharov, L., Laitinen, T., Vainio, R., et al. 2015, ApJ, 806, 80

Kocharov, L., Pesce-Rollins, M., Laitinen, T., et al. 2020, ApJ, 890, 13

Kocharov, L., Vainio, R., Pomoell, J., et al. 2012, ApJ, 753, 87

Kong, X., Gou, F., Giacalone, J., Li, H., \& Chen, Y. 2017, ApJ, 851, 38

Kong, X., Gou, F., Shen, C., et al. 2019, ApJL, 887, L37

Kouloumvakos, A., Rouillard, A., Share, G. H., et al. 2020, ApJ, 893, 76

Kunow, H., Wibberenz, G., Green, G., Müller-Mellin, R., \& Kallenrode, M.-B. 1991, in Physics of Inner Heliosphere, ed. R. Schwenn \& E. Marsch, Vol. 2 (Berlin: Springer), 262
Lee, J.-O., Cho, K.-S., Lee, K.-S., et al. 2020, ApJ, 892, 129

Lemen, J. R., Title, A. M., Akin, D. J., et al. 2012, SoPh, 275, 17

Li, X., Guo, F., Li, H., \& Li, S. 2018, ApJ, 866, 4

Lin, R. P., Dennis, B. R., \& Hurford, G. J. 2002, SoPh, 210, 3

Liu, W., Jin, M., Downs, C., et al. 2018, ApJL, 864, L24

Mandzhavidze, N., \& Ramaty, R. 1992, ApJ, 389, 739

Mandzhavidze, N., Ramaty, R., Bertsch, D. L., \& Schneid, E. J. 1996, in AIP Conf. Proc. 374, High Energy Solar Physics, ed. R. Ramaty, N. Mandzhavidze, \& X.-M. Hua (Melville, NY: AIP), 225

Masson, S., Klein, K.-L., Bütikofer, R., et al. 2009, SoPh, 257, 305

Masuda, S., Kosugi, T., Hara, H., Tsuneta, S., \& Ogawara, Y. 1994, Natur, 371,495

Mishev, A., Usoskin, I., Raukonen, O., et al. 2018, SoPh, 293, 136

Morosan, D. E., Carley, E. P., Hayes, L. A., et al. 2019, NatAs, 3, 452

Murphy, R. J., Ramaty, R., Kozlovsky, B., \& Reames, D. V. 1991, ApJ, 371,793

Omodei, N., Pesce-Rollins, M., Longo, F., Allafort, A., \& Krucker, S. 2018 ApJ, 865, L7

Ramaty, R., Lerners, G., Mandzhavidze, N., \& Miller, J. A. 2000, in AIP Conf. Proc. 528, Acceleration and Transport of Energetic Particles Observed in the Heliosphere: ACE 2000 Symp., ed. R. A. Mewaldt et al. (Melville, NY: AIP), 119

Reames, D. V. 1999, SSRv, 90, 413

Reeves, K. K., Polito, V., Chen, B., et al. 2020, ApJ, 905, 165

Ryan, J. 2000, SSRv, 93, 581

Somov, B. V., \& Kosugi, T. 1997, ApJ, 485, 859

Trottet, G., Samwel, S., Klein, K.-L., Dudok de Wit, T., \& Miteva, R. 2015, SoPh, 290, 819

Vainio, R., Kocharov, L., \& Laitinen, T. 2000, ApJ, 528, 1015

Warren, H., Brooks, D. H., Ugarte-Urra, I., et al. 2018, ApJ, 854, 122

Yu, S., Chen, B., Reaves, K., et al. 2020, ApJ, 900, 17

Zharkova, V. V., Arzner, K., Benz, A. O., et al. 2011, SSRv, 159, 357 